\title{
Trapping and instability of directional gravity waves in localized water currents
}

\author{
B. Eliasson ${ }^{1, *}$ and F. Haas ${ }^{2, \dagger}$ \\ ${ }^{1}$ SUPA, Physics Department, University of Strathclyde, Glasgow G4 ONG, Scotland, United Kingdom \\ ${ }^{2}$ Physics Institute, Universidade Federal do Rio Grande do Sul, CEP 91501-970, Porto Alegre, RS, Brazil
}

(Received 26 September 2013; published 23 June 2014)

\begin{abstract}
The influence of localized water currents on the nonlinear dynamics and stability of large amplitude, statistically distributed gravity waves is investigated theoretically and numerically by means of an evolution equation for a Wigner function governing the spectrum of waves. It is shown that water waves propagating in the opposite direction of a localized current channel can be trapped in the channel, which can lead to the amplification of the wave intensity. Under certain conditions the wave intensity can be further localized due to a self-focusing (Benjamin-Feir) instability. The localized amplification of the wave intensity may increase the probability of extreme events in the form of freak waves, which have been observed in connection with ocean currents.
\end{abstract}

DOI: 10.1103/PhysRevE.89.063014

PACS number(s): 47.35.Bb, 92.10.Hm

\section{INTRODUCTION}

Water currents are often associated with the occurrence of rogue waves on the ocean. For example, several observations of rogue waves have been made off the coasts of South Africa, Japan, and other places associated with strong water currents [1]. Localized water currents also naturally occur at the mouths of big rivers flowing into the oceans or lakes. Field observations of the trapping of water waves in opposing currents have been made in the Gulf stream [2]. Water waves encountering a counterpropagating current will slow down and their wave energy pile up, enhancing their amplitudes and increasing the statistical risk of extreme events. Opposing currents can lead to the steepening and breaking of waves [3], as well as to instabilities and localization of waves [4]. Variable currents can also focus waves into caustic regions [5,6] and capture waves into localized, opposing currents [7-10], where large amplitude waves are produced. There is sometimes a distinction made between extreme waves meaning large in absolute terms, and freak waves meaning unusual waves [5,9]. Naturally occurring waves on the ocean contain a mixture of different frequencies and propagation directions. Statistical descriptions of water waves have been derived in the past using dimensional arguments and by parametrization of experimental data [11,12]. The most widely used kinetic models that govern collective interactions between a spectrum of water waves are Hasselmann's [13] and Zakharov's [14] models for random waves. Hasselmann's equation has been used to model the trapping and intensification of water waves in currents $[2,8,15]$. Alber's model $[16,17]$ uses ideas from quantum statistics [18] to derive a transport model for narrow-banded wave trains. Similar approaches of quantumlike transport equations are used for nonlinear optics [19], plasmas [20], quantum mechanics [21], etc., and are therefore of general interest to nonlinear wave systems. A finite width of the wave spectrum tends to decrease the instability due to phase mixing of the waves, an effect analogous to Landau damping of waves in plasmas [16]. The purpose of this paper is to develop an evolution equation for the Wigner function

\footnotetext{
*bengt.eliasson@strath.ac.uk

${ }^{\dagger}$ fernando.haas@ufrgs.br
}

governing the spectrum of water waves. The model includes the effect of a localized current, in addition to the effects of wave dispersion and nonlinearity, for a distribution of water waves. Using realistic parameters from observations of ocean waves and laboratory experiments, the effects of water currents with different velocities on the wave spectrum are studied numerically.

\section{MATHEMATICAL FORMULATION}

As a starting point, we consider a quasimonochromatic water wave propagating in the $x$ direction with the carrier wave number $k_{0}$ and frequency $\omega_{0}=\sqrt{g k_{0}}$, where $g \approx 9.81 \mathrm{~m} / \mathrm{s}^{2}$ is the gravitational constant (and $k_{0}>0$ ). To model waves with wave vectors and frequencies not too far from the carrier wave, taking into account first-order dispersive and nonlinear effects, as well as the influence of a background water flow, we assume that the surface elevation is given by $\eta(x, y, t)=(1 / 2) A(x, y, t) \exp \left(-i \omega_{0} t+i k_{0} x\right)+$ complex conjugate, where $A$ is the complex-valued, slowly varying envelope of the wave. To take into account the effects of a water current localized in the $y$ direction and flowing in the $x$ direction (cf. Fig. 1), we use as a starting point the nonlinear Schrödinger equation

$$
\begin{aligned}
& i\left(\frac{\partial A}{\partial t}+v_{0}(y) \frac{\partial A}{\partial x}\right)-k_{0} v_{\mathrm{c}}(y) A+D_{x} \frac{\partial^{2} A}{\partial x^{2}} \\
& \quad+D_{y} \frac{\partial^{2} A}{\partial y^{2}}-\xi|A|^{2} A=0,
\end{aligned}
$$

where $D_{x}=-\omega_{0} /\left(8 k_{0}^{2}\right)$ and $D_{y}=\omega_{0} /\left(4 k_{0}^{2}\right)$ are the group dispersion coefficients, $v_{0}(y)=v_{\mathrm{gr}}+v_{\mathrm{c}}(y)$ is the sum of the group velocity $v_{\mathrm{gr}}=\partial \omega / \partial k_{0}=\omega_{0} /\left(2 k_{0}\right)$ and water current velocity $v_{\mathrm{c}}(y)$, and $\xi=\omega_{0} k_{0}^{2} / 2$ is the nonlinear coupling constant. The term $v_{0}(y) \partial A / \partial x$ takes into account the modification of the effective group velocity due to the water current, and the term $-k_{0} v_{\mathrm{c}}(y) A$ governs the frequency Doppler shift due to the current. Higher-order expansions in terms of nonlinearity and wave dispersion have been used to derive more general Schrödinger-like models for water waves including currents [22]. In the formalism of Ref. [22], the current velocity is of order $\epsilon^{2}$, while the time and space derivatives and $A k_{0}$ are of order $\epsilon$, where $\epsilon$ is a nonlinearity 


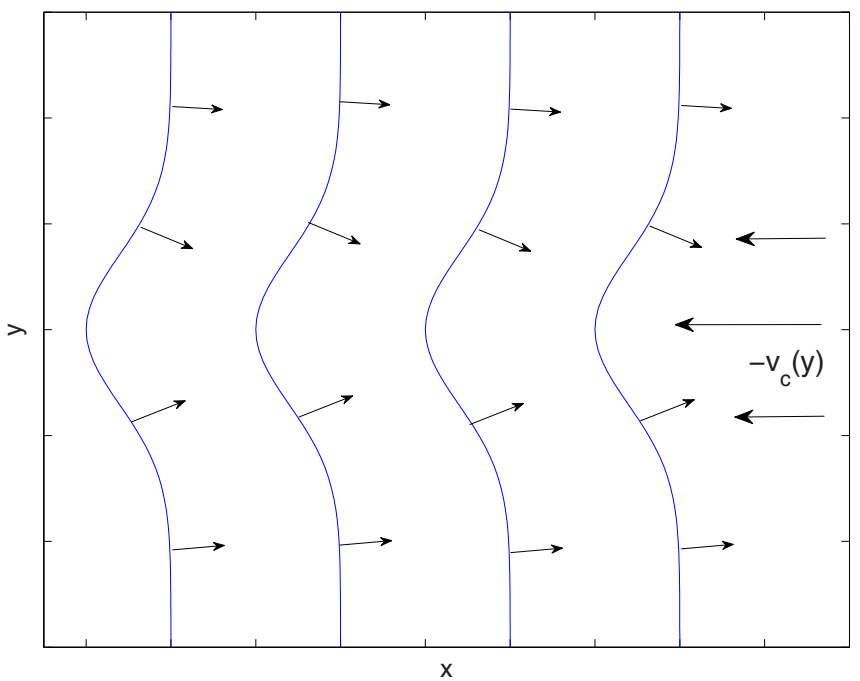

FIG. 1. (Color online) Localized water current with velocity $v_{c}(y)$ opposite to the wave direction, leading to the bending of the wave fronts and an accumulation of water waves in the channel.

parameter. Since the dispersion and nonlinearity coefficients $D_{x}$ and $-\xi$ have equal signs, Eq. (1) is modulationally unstable due to the Benjamin-Feir instability (BFI) for modulation wave numbers in the forward $x$ direction but is stable in the perpendicular $y$ direction, since $D_{y}$ and $-\xi$ have opposite signs. The idea of trapping of water waves in a localized flow current [10] can be emphasized by the reduced problem where $A$ depends only on $y$ and $t$ (but not on $x$ ),

$$
i \frac{\partial A}{\partial t}+D_{y} \frac{\partial^{2} A}{\partial y^{2}}-V(y) A-\xi|A|^{2} A=0,
$$

where $V(y)=k_{0} v_{\mathrm{c}}(y)$ works as an effective potential. This is formally equivalent to the one-dimensional Gross-Pitaevskii equation (e.g., Ref. [23]), used to model Bose-Einstein condensates with a repulsive coupling coefficient (since $\xi>0$ ). Under suitable conditions, where the localized water current is opposite to the direction of the waves [i.e., when $v_{\mathrm{c}}(y)$ is negative], water waves can be trapped in the potential well $V(y)$. A linearized version of Eq. (2) leads to a SturmLiouville type boundary-value problem supporting both a discrete spectrum corresponding to the trapped waves and a continuous spectrum corresponding to free waves. Hence, in generic situations, there is a combination of trapped and free waves [10]. The nonlinearity is defocusing in the $y$ direction and decreases the share of the trapped component. On the other hand, the trapping and accumulation of waves can enhance the growth rate of the BFI in the channel when the $x$ dependence is taken into account.

Instead of the highly idealized model using monochromatic water waves, it is interesting to investigate the dynamics of a spectrum of waves which is more realistic for real gravity waves. To derive an evolution equation for the wave spectrum, we define the Wigner function,

$f=f(\mathbf{r}, \mathbf{v}, t)=\frac{1}{2(2 \pi)^{2}} \int A^{*}(+) A(-) e^{i \lambda \cdot\left[\mathbf{v}-v_{0}(y) \hat{\mathbf{x}}\right]} d^{2} \lambda$, where we used the notation $A( \pm)=A\left(\mathbf{R}_{ \pm}, t\right)$ and $A^{*}( \pm)=$ $A^{*}\left(\mathbf{R}_{ \pm}, t\right)$, with $\mathbf{R}_{ \pm}=\left(x_{ \pm}, y_{ \pm}\right)=\left(x \pm D_{x} \lambda_{x}, y \pm D_{y} \lambda_{y}\right)$. The velocity variable $\mathbf{v}=v_{x} \hat{\mathbf{x}}+v_{y} \hat{\mathbf{y}}$ can be interpreted as the group velocity with which the energy of each wave is transported, and $\lambda=\lambda_{x} \hat{\mathbf{x}}+\lambda_{y} \hat{\mathbf{y}}$ is the Fourier transformed velocity variable, where $\hat{\mathbf{x}}$ and $\hat{\mathbf{y}}$ are the unit vectors along the $x$ and $y$ directions. The wave intensity (the variance of the surface elevation) is defined as $I=\int f(\mathbf{r}, \mathbf{v}, t) d^{2} v$. If the wave spectrum is entirely defined by a deterministic wave train $A$, we have from Eq. (3) that $I=|A|^{2} / 2$. Following Alber [16], however, we use ideas from statistical mechanics to describe the dynamics of an ensemble of waves and to abandon the deterministic view. The Wigner function (3) is then used to generate an evolution equation, which contains the velocity $\mathbf{v}$ as a variable in addition to space and time [24]. Taking the time derivative of both sides of Eq. (3) and eliminating the time derivatives of $A$ and $A^{*}$ with the help of Eq. (1), we derive the evolution equation for the Wigner function

$$
\begin{aligned}
\frac{\partial f}{\partial t}+\mathbf{v} \cdot \nabla f= & \frac{1}{(2 \pi)^{2}} \int\left\{2 i \xi[I(+)-I(-)]+i\left[v_{\mathrm{c}}\left(y_{+}\right)\right.\right. \\
& \left.-v_{\mathrm{c}}\left(y_{-}\right)\right]\left[k_{0}-\frac{\left(v_{0}(y)-v_{x}\right)}{2 D_{x}}\right] \\
& \left.-\frac{\left[v_{\mathrm{c}}\left(y_{+}\right)-2 v_{\mathrm{c}}(y)+v_{\mathrm{c}}\left(y_{-}\right)\right]}{2} \frac{\partial}{\partial x}\right\} e^{i \lambda \cdot\left(\mathbf{v}-\mathbf{v}^{\prime}\right)} \\
& \times f\left(\mathbf{r}, \mathbf{v}^{\prime}, t\right) d^{2} v^{\prime} d^{2} \lambda-v_{y} \frac{\partial v_{\mathrm{c}}(y)}{\partial y} \frac{\partial f}{\partial v_{x}},
\end{aligned}
$$

where the wave intensity is $I=\int f(\mathbf{r}, \mathbf{v}, t) d^{2} v$. A detailed derivation of Eq. (4) is given in the Appendix. For zero current, $v_{c}=0$, Eq. (4) can be shown to be equivalent to Eq. (3.14) of Ref. [16] in the deep water limit. After integrating Eq. (4) in velocity space, we find the continuity equation

$$
\frac{\partial I}{\partial t}+\nabla \cdot \int \mathbf{v} f(\mathbf{r}, \mathbf{v}, t) d^{2} v=0,
$$

which shows that the total wave energy $\mathcal{E}=\int I d^{2} r$ is conserved.

A model spectrum in frequency domain, based on the Pierson-Moskowitz spectrum [11], was parametrized by the Joint North Sea Wave Project (JONSWAP) [12] as

$$
S(\omega)=\frac{\alpha_{P} g^{2}}{\omega^{5}} \exp \left(-\frac{5}{4} \frac{\omega_{p}^{4}}{\omega^{4}}\right) \gamma^{\exp \left[-\frac{\left(\omega-\omega_{p}\right)^{2}}{2 \sigma^{2} \omega_{p}^{2}}\right]},
$$

where $\omega_{p}$ is the peak frequency, $\gamma$ is the peak enhancement parameter, and $\alpha_{P}$ is the Phillips parameter. Since the wave spectrum is peaked at $\omega=\omega_{p}$, we will use $\omega_{0}=\omega_{p}$ and $k_{0}=$ $k_{p} \equiv \omega_{p}^{2} / g$ in the evaluation of the dispersion coefficients $D_{x}$ and $D_{y}$. The integral of the spectrum $S(\omega)$ in Eq. (6) over all frequencies yields the wave intensity. To use the directional JONSWAP spectrum to construct initial conditions in our simulations, we will use $f=f_{0}(\mathbf{v})=F_{0}(v) G(\theta)$, where we have introduced polar coordinates $v_{x}=v \cos (\theta)$ and $v_{y}=v \sin (\theta)$ in velocity space. We obtain $F_{0}$ from the frequency spectrum (6) by using the differential variance $d I=S(\omega) d \omega=F_{0}(v) v d v$, as

$$
F_{0}(v)=S[\omega(v)] \frac{1}{v}\left|\frac{d \omega}{d v}\right|=S[\omega(v)] \frac{g}{2 v^{3}},
$$


where we used that the group speed $v$ of the wave packets is related to the wave frequency $\omega=\sqrt{g k}$ via $v=d \omega / d k=$ $\omega / 2 k=g / 2 \omega$, or $\omega(v)=g / 2 v$. The directional spreading function is chosen [25,26] as $G(\theta)=G_{0} \cos ^{2 s}(\theta / 2)=$ $G_{0}[1+\cos (\theta)]^{s} / 2^{s}$, where $\cos (\theta)=v_{x} / v, v=\left(v_{x}^{2}+v_{y}^{2}\right)^{1 / 2}$, and $G_{0}=2^{2 s-1} \Gamma^{2}(s+1) /[\pi \Gamma(2 s+1)]$ is a normalization constant [26] such that $\int_{-\pi}^{\pi} G(\theta) d \theta=1$, where $\Gamma$ is the Gamma function and $s$ is a directional spread parameter. We note that $G$ has a maximum at $\theta=0$ and tends to a narrower distribution with an increase of $s$. For ocean waves [12], the parameter $\gamma$ is in the range $1-6$ and $\alpha_{P}$ in the range $0.01-0.05$; the values $\gamma=1$ and $\alpha_{P}=0.0081$ give Pierson-Moskowitz spectrum [11] of fully developed wind seas. Typical values on the directional spread parameter $s$ is of the order 5-20 for typical ocean waves [26,27], but larger values (corresponding to more unidirectional waves) can be obtained in controlled experiments [28].

For numerical convenience and for comparison with Alber's model [16], we Fourier transform Eq. (4) in velocity space. Using the Fourier transform pair

$$
\begin{aligned}
\widehat{f}(\mathbf{r}, \lambda, t) & =2 \int f(\mathbf{r}, \mathbf{v}, t) e^{i \lambda \cdot \mathbf{v}} d^{2} v \\
f(\mathbf{r}, \mathbf{v}, t) & =\frac{1}{2(2 \pi)^{2}} \int \widehat{f}(\mathbf{r}, \lambda, t) e^{-i \lambda \cdot \mathbf{v}} d^{2} \lambda
\end{aligned}
$$

in Eq. (4) leads to

$$
\begin{aligned}
\frac{\partial \widehat{f}}{\partial t}-i \nabla_{\lambda} \cdot \nabla \widehat{f}= & -\left\{2 i \xi[I(+)-I(-)]+i\left[v_{\mathrm{c}}\left(y_{+}\right)\right.\right. \\
& \left.-v_{\mathrm{c}}\left(y_{-}\right)\right]\left[k_{0}-\frac{1}{2 D_{x}}\left(v_{0}(y)+i \frac{\partial}{\partial \lambda_{x}}\right)\right] \\
& \left.+\frac{\left[v_{\mathrm{c}}\left(y_{+}\right)-2 v_{\mathrm{c}}(y)+v_{\mathrm{c}}\left(y_{-}\right)\right]}{2} \frac{\partial}{\partial x}\right\} \widehat{f} \\
& +\lambda_{x} \frac{\partial v_{\mathrm{c}}(y)}{\partial y} \frac{\partial \widehat{f}}{\partial \lambda_{y}}
\end{aligned}
$$

where $I=\widehat{f}(\mathbf{r}, 0, t) / 2$. Compared to Eq. (3.7) of Ref. [16], Eq. (9) also includes the effects of the water current $v_{c}$.

\section{NUMERICAL RESULTS AND DISCUSSION}

To solve Eq. (9) numerically, we employ the methods in Ref. [29], originally developed to solve the Vlasov equation for plasmas. Using a pseudospectral method in space, the spatial shifts are transformed to multiplications in wave-number space, which are readily evaluated numerically (cf. Ref. [24]). As initial conditions we use the JONSWAP spectrum with homogeneously distributed water waves. Equation (8a) is evaluated numerically to obtain the initial distribution function in the Fourier transformed velocity space. The used parameters $\gamma=6, \sigma=0.08$, and $s=20$ are consistent with JONSWAP measurements [12] and recent water basin experiments [28]. For $\alpha_{P}=0.05$, the initial wave intensity is $I \approx 0.01 k_{0}^{-2}$ [cf. Fig. 2(a)]. Small random fluctuations of the order $2 \times 10^{-5} k_{0}^{-2}$ are added to the initial intensity to give a seed to the BFI. The velocity profile of the water current, flowing in the $x$ direction, is given by $v_{c}(y)=v_{c 0} \exp \left(-y^{2} / L^{2}\right)$, where $L$ is the width of the current channel. For numerical convenience,

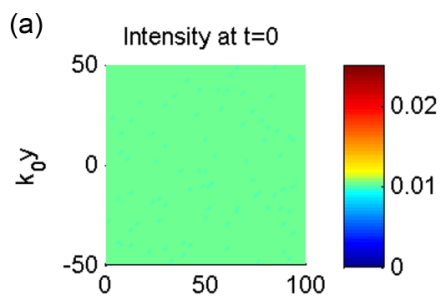

(c)

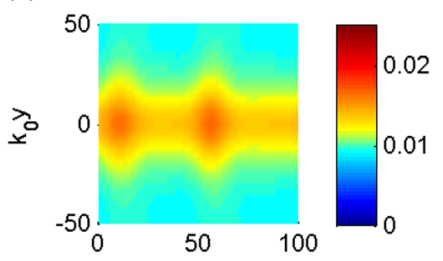

(e)

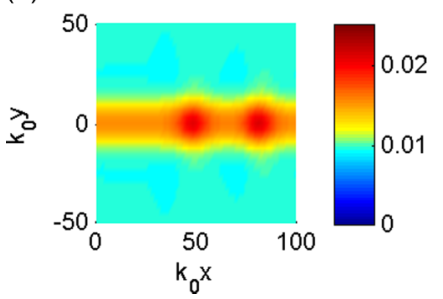

(b)

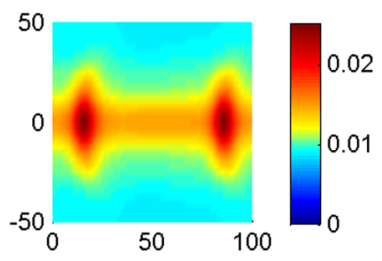

(d)

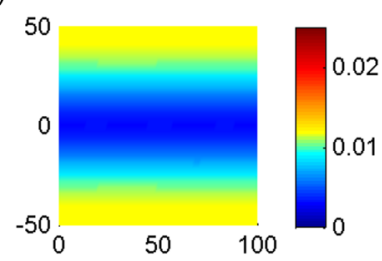

(f)

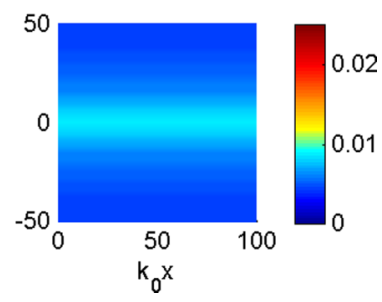

FIG. 2. (Color online) Wave intensity for different amplitudes and signs of the water current flowing in the $x$ direction, given by $v_{c}(y)=v_{c 0} \exp \left(-y^{2} / L^{2}\right)$. (a) The initial $(t=0)$ intensity $I \approx$ $0.01 k_{0}^{-2}$ for $\alpha_{P}=0.05$, with small (of the order $2 \times 10^{-5} k_{0}^{-2}$ ) random fluctuations added to seed the BFI. (b)-(f) The intensities at $t=3800 T_{0}$ for (b) $v_{c 0}=-0.08 v_{\mathrm{ph}}, \alpha_{P}=0.05, L=20 k_{0}^{-1}$, (c) $v_{c 0}=-0.04 v_{\mathrm{ph}}, \alpha_{P}=0.05, L=20 k_{0}^{-1}$, (d) $v_{c 0}=+0.08 v_{\mathrm{ph}}$, $\alpha_{P}=0.05, L=20 k_{0}^{-1}$, (e) $v_{c 0}=-0.08 v_{\mathrm{ph}}, \alpha_{P}=0.05, L=10 k_{0}^{-1}$, and (f) $v_{c 0}=-0.08 v_{\mathrm{ph}}, \alpha_{P}=0.025, L=20 k_{0}^{-1}$, where $v_{\mathrm{ph}}=\omega_{0} / k_{0}$ is the phase velocity of the leading wave. The wave energy is localized by opposing currents (negative $v_{c 0}$ ) and dispersed by following currents (positive $v_{c 0}$ ). Wave localizations due to the BFI are seen for the opposing currents in panels (b), (c), and (e).

the simulations are carried out in a window centered around the spectral peak of initial distribution $f_{0}(\mathbf{v})$, by transforming to a frame moving in the $x$ direction with the group velocity $v_{\text {gr }}=v_{\mathrm{ph}} / 2$, where $v_{\mathrm{ph}}=\omega_{0} / k_{0}$ is the phase speed of the leading wave, i.e., $v_{x} \rightarrow v_{x}+v_{\text {gr }}$ in the initial condition and in Eq. (4), $\partial / \partial t \rightarrow \partial / \partial t-v_{\text {gr }} \partial / \partial x$ in Eq. (4), and $\partial / \partial \lambda_{x} \rightarrow$ $\partial / \partial \lambda_{x}+i v_{\text {gr }}$ and $\partial / \partial t \rightarrow \partial / \partial t-v_{\text {gr }} \partial / \partial x$ in Eq. (9). The spatial domain of size $L_{x} \times L_{y}=100 \times 200 k_{0}^{-1}$ is resolved by $64 \times 64$ grid points, with periodic boundary conditions. The velocity space of size $1.0 \times 1.0 v_{\text {ph }}$ is resolved using $80 \times 80$ intervals. (The corresponding domain in Fourier transformed velocity space is $80 \pi \times 80 \pi v_{\text {ph }}^{-1}$ [29].)

Figures 2(b)-2(f) show the intensity distributions at the end of the simulations at $t=3800 T_{0}$ for different values of $v_{c 0}, \alpha_{P}$, and $L$, where $T_{0}=2 \pi / \omega_{0}$ is the wave period of the leading wave. It is seen in Fig. 2 that opposing currents $\left(v_{c 0}<0\right)$ attract surrounding waves and the intensity is increased at the center of the channel. Since the total wave energy is conserved [cf. Eq. (5)], an increase of the intensity in the channel leads to a slight decrease of the intensity at the transverse periphery, and vice versa. For the opposing current $v_{c 0}=-0.08 v_{\mathrm{ph}}$ in 
a channel of width $L=20 k_{0}^{-1}$ [Fig. 2(b)], the wave energy is further amplified due to the BFI. The instability saturates nonlinearly by the formation of long-lived, localized wave packets in the center of the channel. For smaller values of the opposing current [Fig. 2(c)] the intensity (and the resulting instability) in the channel is decreased, and for a following current $\left[v_{c 0}>0\right.$ in Fig. 2(d)] the waves are instead repelled, resulting in a minimum of the intensity at the center of the channel. A decrease of the width of the channel [Fig. 2(e)] only slightly decreases the intensity in the channel. Finally, decreasing the initial intensity by half using $\alpha_{P}=0.025$ [cf. Fig. 2(f)] leads to a stabilization of the system. The overall maximum of the intensity as a function of time is shown in Fig. 3. The intensity has some initial transient oscillations when the waves are redistributed from the homogeneous initial condition to be either trapped in or repelled by the channel. Due to the trapping of waves, the intensity is higher for higher opposing currents, and a further increase of the maximum intensity occurs due to the BFI for cases (b), (c), and (e) with opposing currents. We emphasize that the numerical results here apply to both ocean waves and experiment using water tanks or basins. For example, for ocean waves having the frequency $0.1 \mathrm{~Hz}$ and corresponding angular frequency $\omega=0.628 \mathrm{~s}^{-1}$, wave number $k_{0}=0.040 \mathrm{~m}^{-1}$, and phase speed $v_{\mathrm{ph}}=16 \mathrm{~m} / \mathrm{s}$, the flow speed used in Fig. 2(b) is $v_{c 0}=$ $-0.08 v_{\mathrm{ph}} \approx-1.28 \mathrm{~m} / \mathrm{s}$ at the center of the channel. A width of the current channel of $L=20 k_{0}^{-1}$ corresponds to $500 \mathrm{~m}$ for this case, to be compared with the wavelength $2 \pi / k_{0} \approx 160 \mathrm{~m}$ for the leading wave. On the other hand, for experiments [28] using waves with a frequency $1 \mathrm{~Hz}$, corresponding to $\omega=6.28 \mathrm{~s}^{-1}$, $k_{0}=4.0 \mathrm{~m}^{-1}$, and $v_{\mathrm{ph}}=1.6 \mathrm{~m} / \mathrm{s}$, we instead have a flow speed of $v_{c 0}=-0.08 v_{\mathrm{ph}} \approx-12.8 \mathrm{~cm} / \mathrm{s}$ at the center of the channel with a width of $L=20 k_{0}^{-1}=5 \mathrm{~m}$.

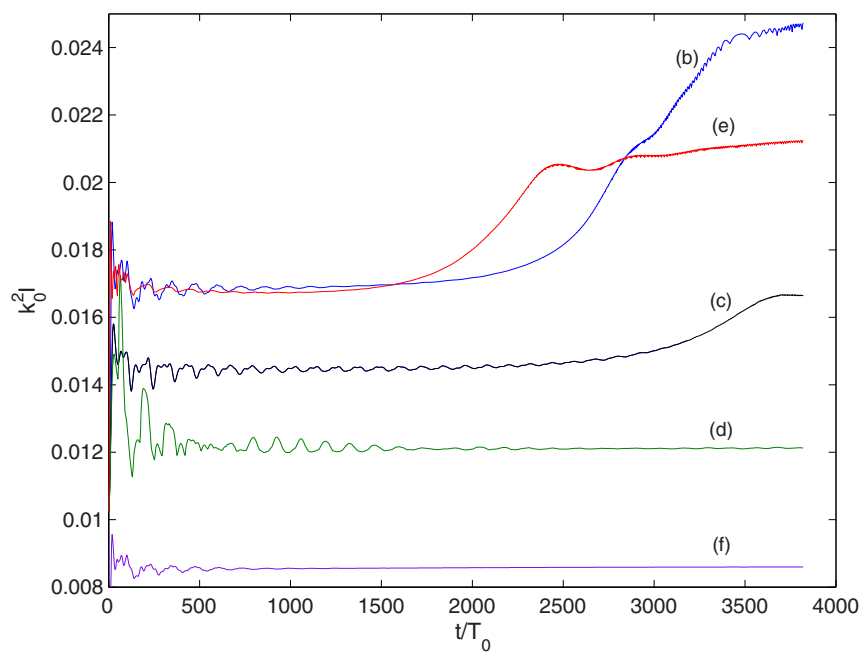

FIG. 3. (Color online) Maximum wave intensity as a function of time. The labels (b)-(f) refer to the different cases in Fig. 2).

In summary, localized opposing water currents trap water waves which via the BFI can lead to the formation of long-lived, large amplitude wave packets propagating along the channel. This may enhance the statistical probability of extreme events in the form of giant waves.

\section{ACKNOWLEDGMENTS}

F.H. acknowledges the support by Conselho Nacional de Desenvolvimento Científico e Tecnológico (CNPq).

\section{APPENDIX: DERIVATION OF THE EVOLUTION EQUATION FOR THE WIGNER FUNCTION}

As a starting point to derive Eq. (4), we consider the nonlinear Schrödinger equation

$$
i\left(\frac{\partial A}{\partial t}+v_{0}(y) \frac{\partial A}{\partial x}\right)-k_{0} v_{c}(y) A+D_{x} \frac{\partial^{2} A}{\partial x^{2}}+D_{y} \frac{\partial^{2} A}{\partial y^{2}}-\xi|A|^{2} A=0,
$$

where

$$
D_{x}=-\frac{\omega_{0}}{8 k_{0}^{2}}, \quad D_{y}=\frac{\omega_{0}}{4 k_{0}^{2}}
$$

are the group dispersion coefficients, $v_{0}(y)=v_{\mathrm{gr}}+v_{c}(y)$ is the sum of the group velocity $v_{\mathrm{gr}}=\partial \omega / \partial k_{0}=\omega_{0} /\left(2 k_{0}\right)$ and the water current velocity $v_{c}(y)$, which introduces a $y$-dependent Doppler shift in the nonlinear Schrödinger equation, and $\xi=\omega_{0} k_{0}^{2} / 2$ is the nonlinear coupling constant. We define the Wigner function

$$
f=f(\mathbf{r}, \mathbf{v}, t)=\frac{1}{2(2 \pi)^{2}} \int d^{2} \lambda e^{i \lambda \cdot\left[\mathbf{v}-v_{0}(y) \hat{\mathbf{x}}\right]} A^{*}\left(\mathbf{R}_{+}, t\right) A\left(\mathbf{R}_{-}, t\right),
$$

where $\mathbf{R}_{+}=\left(x_{+}, y_{+}\right)=\left(x+D_{x} \lambda_{x}, y+D_{y} \lambda_{y}\right), \mathbf{R}_{-}=\left(x_{-}, y_{-}\right)=\left(x-D_{x} \lambda_{x}, y-D_{y} \lambda_{y}\right)$, and the asterisk denotes complex conjugation. Taking the time derivative of both sides of Eq. (A3) and replacing time derivatives of $A$ and $A^{*}$ on the right-hand side with the help of Eq. (A1) gives

$$
\frac{\partial f}{\partial t}=I_{1}+I_{2}+I_{3}+I_{4}
$$

where

$$
I_{1}=\frac{1}{2(2 \pi)^{2}} \int d^{2} \lambda e^{i \lambda \cdot\left[\mathbf{v}-v_{0}(y) \hat{\mathbf{x}}\right]}\left(v_{0}\left(y_{+}\right) \frac{\partial A^{*}(+)}{\partial x} A(-)+v_{0}\left(y_{-}\right) A^{*}(+) \frac{\partial A(-)}{\partial x}\right)
$$




$$
\begin{gathered}
I_{2}=\frac{i k_{0}}{2(2 \pi)^{2}} \int d^{2} \lambda e^{i \lambda \cdot\left[\mathbf{v}-v_{0}(y) \hat{\mathbf{x}}\right]}\left[v_{c}\left(y_{+}\right)-v_{c}\left(y_{-}\right)\right] A^{*}(+) A(-) \\
=\frac{i k_{0}}{(2 \pi)^{2}} \int d^{2} \lambda d^{2} v^{\prime} e^{i \lambda \cdot\left(\mathbf{v}-\mathbf{v}^{\prime}\right)}\left[v_{c}\left(y_{+}\right)-v_{c}\left(y_{-}\right)\right] f\left(\mathbf{r}, \mathbf{v}^{\prime}, t\right), \\
I_{3}=\frac{i}{2(2 \pi)^{2}} \int d^{2} \lambda e^{i \lambda \cdot\left[\mathbf{v}-v_{0}(y) \hat{\mathbf{x}}\right]}\left[D_{x}\left(A^{*}(+) \frac{\partial^{2} A(-)}{\partial x^{2}}-\frac{\partial^{2} A^{*}(+)}{\partial x^{2}} A(-)\right)+D_{y}\left(A^{*}(+) \frac{\partial^{2} A(-)}{\partial y^{2}}-\frac{\partial^{2} A^{*}(+)}{\partial y^{2}} A(-)\right)\right] \\
=-\left[v_{x}-v_{0}(y)\right] \frac{\partial f}{\partial x}-v_{y} \frac{\partial f}{\partial y}-v_{y} \frac{\partial v_{0}(y)}{\partial y} \frac{\partial f}{\partial v_{x}}, \\
I_{4}=\frac{i \xi}{2(2 \pi)^{2}} \int d^{2} \lambda e^{i \lambda \cdot\left[\mathbf{v}-v_{0}(y) \hat{\mathbf{x}}\right]}\left[|A(+)|^{2}-|A(-)|^{2}\right] A^{*}(+) A(-)=\frac{2 i \xi}{(2 \pi)^{2}} \int d^{2} \lambda d^{2} v^{\prime} e^{i \lambda \cdot\left(\mathbf{v}-\mathbf{v}^{\prime}\right)}[I(+)-I(-)] f\left(\mathbf{r}, \mathbf{v}^{\prime}, t\right),
\end{gathered}
$$

where the notation $A\left(\mathbf{R}_{ \pm}, t\right)=A( \pm)$ and $A^{*}\left(\mathbf{R}_{ \pm}, t\right)=A^{*}( \pm)$ is used.

In the derivation of Eq. (A6), the identity

$$
\int d^{2} v e^{-i \lambda \cdot\left[\mathbf{v}-v_{0}(y) \hat{\mathbf{x}}\right]} f(\mathbf{r}, \mathbf{v}, t)=\frac{1}{2} A^{*}(+) A(-)
$$

was used.

In the derivation of Eq. (A7), the identities

$$
\begin{aligned}
& \frac{\partial^{2}}{\partial x \partial \lambda_{x}}\left[A^{*}(+) A(-)\right]=D_{x}\left(\frac{\partial^{2} A^{*}(+)}{\partial x^{2}} A(-)-A^{*}(+) \frac{\partial^{2} A(-)}{\partial x^{2}}\right), \\
& \frac{\partial^{2}}{\partial y \partial \lambda_{y}}\left[A^{*}(+) A(-)\right]=D_{y}\left(\frac{\partial^{2} A^{*}(+)}{\partial y^{2}} A(-)-A^{*}(+) \frac{\partial^{2} A(-)}{\partial y^{2}}\right)
\end{aligned}
$$

were used, as well as integration by parts assuming decaying or periodic boundary conditions.

In the derivation of Eq. (A8), the quantity

$$
I(\mathbf{r}, t)=\int d^{2} v f(\mathbf{r}, \mathbf{v}, t)=\frac{1}{2}|A(\mathbf{r}, t)|^{2}
$$

was introduced, and the identity (A9) was again used.

Hence the main difficulty in evaluating the time-evolution equation for the Wigner distribution is in the integral $I_{1}$ in Eq. (A5). We will use the formal expression

$$
v_{0}\left(y_{ \pm}\right)=v_{0}\left(y \pm D_{y} \lambda_{y}\right)=v_{0}(y) \exp \left( \pm D_{y} \lambda_{y} \frac{\overleftarrow{\partial}}{\partial y}\right)
$$

which can be justified by Taylor expansions of $v_{0}$ and the exponential. The left arrow $\leftarrow$ indicates that $\partial / \partial y$ acts to the left. We have

$$
I_{1}=-\frac{v_{0}(y)}{2(2 \pi)^{2}} \int\left[\exp \left(D_{y} \lambda_{y} \frac{\overleftarrow{\partial}}{\partial y}\right) \frac{\partial A^{*}}{\partial x}(+) A(-)+\exp \left(-D_{y} \lambda_{y} \frac{\overleftarrow{\partial}}{\partial y}\right) A^{*}(+) \frac{\partial A}{\partial x}(-)\right] e^{i \lambda \cdot\left[\mathbf{v}-v_{0}(y) \hat{\mathbf{x}}\right]} d^{2} \lambda
$$

We now use a formal property of the Fourier transform $\lambda_{y} \rightarrow-i \partial / \partial v_{y}$ to write

$$
\int \exp \left(D_{y} \lambda_{y} \frac{\overleftarrow{\partial}}{\partial y}\right) \frac{\partial A^{*}}{\partial x}(+) A(-) e^{i \lambda \cdot\left[\mathbf{v}-v_{0}(y) \hat{\mathbf{x}}\right]} d^{2} \lambda=\exp \left(-i D_{y} \frac{\overleftarrow{\partial}}{\partial y} \frac{\vec{\partial}}{\partial v_{y}}\right) \int \frac{\partial A^{*}}{\partial x}(+) A(-) e^{i \lambda \cdot\left[\mathbf{v}-v_{0}(y) \hat{\mathbf{x}}\right]} d^{2} \lambda
$$

and

$$
\int \exp \left(-D_{y} \lambda_{y} \frac{\overleftarrow{\partial}}{\partial y}\right) A^{*}(+) \frac{\partial A}{\partial x}(-) e^{i \lambda \cdot\left[\mathbf{v}-v_{0}(y) \hat{\mathbf{x}}\right]} d^{2} \lambda=\exp \left(i D_{y} \frac{\overleftarrow{\partial}}{\partial y} \frac{\vec{\partial}}{\partial v_{y}}\right) \int A^{*}(+) \frac{\partial A}{\partial x}(-) e^{i \lambda \cdot\left[\mathbf{v}-v_{0}(y) \hat{\mathbf{x}}\right]} d^{2} \lambda
$$


Inserting Eqs. (A15) and (A16) into Eq. (A14), and writing the exponentials in front of the integral signs in terms of trigonometric functions as $\exp ( \pm i \alpha)=\cos (\alpha) \pm i \sin (\alpha)$, where $\alpha=D_{y}(\overleftarrow{\partial} / \partial y)\left(\vec{\partial} / \partial v_{y}\right)$, we obtain

$$
\begin{aligned}
I_{1}= & -\frac{v_{0}(y)}{2(2 \pi)^{2}}\left\{\cos \left(D_{y} \frac{\overleftarrow{\partial}}{\partial y} \frac{\vec{\partial}}{\partial v_{y}}\right) \int\left[\frac{\partial A^{*}}{\partial x}(+) A(-)+A^{*}(+) \frac{\partial A}{\partial x}(-)\right] e^{i \lambda \cdot\left[\mathbf{v}-v_{0}(y) \hat{\mathbf{x}}\right]} d^{2} \lambda\right. \\
& \left.-i \sin \left(D_{y} \frac{\overleftarrow{\partial}}{\partial y} \frac{\vec{\partial}}{\partial v_{y}}\right) \int\left[\frac{\partial A^{*}}{\partial x}(+) A(-)-A^{*}(+) \frac{\partial A}{\partial x}(-)\right] e^{i \lambda \cdot\left[\mathbf{v}-v_{0}(y) \hat{\mathbf{x}}\right]} d^{2} \lambda\right\} .
\end{aligned}
$$

Finally, we use that

$$
\frac{\partial A^{*}}{\partial x}(+) A(-)+A^{*}(+) \frac{\partial A}{\partial x}(-)=\frac{\partial}{\partial x}\left[A^{*}(+) A(-)\right]
$$

and

$$
\frac{\partial A^{*}}{\partial x}(+) A(-)-A^{*}(+) \frac{\partial A}{\partial x}(-)=\frac{1}{D_{x}} \frac{\partial}{\partial \lambda_{x}}\left[A^{*}(+) A(-)\right]
$$

and an integration by parts in Eq. (A17) to move the derivative with respect to $\lambda_{x}$ from $\left[A^{*}(+) A(-)\right]$ to $e^{i \lambda \cdot\left[\mathbf{v}-v_{0}(y) \hat{\mathbf{x}}\right]}$. The result is

$$
\begin{aligned}
I_{1}= & -\frac{v_{0}(y)}{2(2 \pi)^{2}}\left\{\cos \left(D_{y} \frac{\overleftarrow{\partial}}{\partial y} \frac{\vec{\partial}}{\partial v_{y}}\right) \frac{\partial}{\partial x} \int A^{*}(+) A(-) e^{i \lambda \cdot\left[\mathbf{v}-v_{0}(y) \hat{x}\right]} d^{2} \lambda\right. \\
& \left.-\sin \left(D_{y} \frac{\overleftarrow{\partial}}{\partial y} \frac{\vec{\partial}}{\partial v_{y}}\right) \frac{\left[v_{x}-v_{0}(y)\right]}{D_{x}} \int A^{*}(+) A(-) e^{i \lambda \cdot\left[\mathbf{v}-v_{0}(y) \hat{x}\right]} d^{2} \lambda\right\},
\end{aligned}
$$

or, using the definition (A3) of the Wigner function,

$$
I_{1}=-v_{0}(y) \cos \left[D_{y} \frac{\overleftarrow{\partial}}{\partial y} \frac{\vec{\partial}}{\partial v_{y}}\right] \frac{\partial f}{\partial x}+v_{0}(y) \sin \left[D_{y} \frac{\overleftarrow{\partial}}{\partial y} \frac{\vec{\partial}}{\partial v_{y}}\right] \frac{\left[v_{x}-v_{0}(y)\right]}{D_{x}} f
$$

The expression for $I_{1}$ can be shown in an integral form using the identities

$$
v_{0}(y) \cos \left[D_{y} \frac{\overleftarrow{\partial}}{\partial y} \frac{\vec{\partial}}{\partial v_{y}}\right] \frac{\partial f}{\partial x}=\frac{1}{2(2 \pi)^{2}} \frac{\partial}{\partial x} \int d^{2} \lambda d^{2} v^{\prime} e^{i \lambda \cdot\left(\mathbf{v}-\mathbf{v}^{\prime}\right)}\left[v_{0}\left(y_{+}\right)+v_{0}\left(y_{-}\right)\right] f\left(\mathbf{r}, \mathbf{v}^{\prime}, t\right)
$$

and

$$
v_{0}(y) \sin \left[D_{y} \frac{\overleftarrow{\partial}}{\partial y} \frac{\vec{\partial}}{\partial v_{y}}\right] \frac{\left[v_{x}-v_{0}(y)\right]}{D_{x}} f=\frac{i}{2(2 \pi)^{2}} \frac{\left[v_{x}-v_{0}(y)\right]}{D_{x}} \int d^{2} \lambda d^{2} v^{\prime} e^{i \lambda \cdot\left(\mathbf{v}-\mathbf{v}^{\prime}\right)}\left[v_{0}\left(y_{+}\right)-v_{0}\left(y_{-}\right)\right] f\left(\mathbf{r}, \mathbf{v}^{\prime}, t\right),
$$

which can be proven after Taylor expanding and using the definition of the Wigner function. In this way we arrive at the form

$$
I_{1}=\frac{1}{2(2 \pi)^{2}} \int d^{2} \lambda d^{2} v^{\prime} e^{i \lambda \cdot\left(\mathbf{v}-\mathbf{v}^{\prime}\right)}\left[-\left[v_{0}\left(y_{+}\right)+v_{0}\left(y_{-}\right)\right] \frac{\partial}{\partial x}+\frac{i\left[v_{x}-v_{0}(y)\right]}{D_{x}}\left[v_{0}\left(y_{+}\right)-v_{0}\left(y_{-}\right)\right]\right] f\left(\mathbf{r}, \mathbf{v}^{\prime}, t\right) .
$$

To conclude, we use the results (A6), (A7), (A8), and (A24) and the definition of group velocity, $v_{\mathrm{gr}}=v_{0}(y)-v_{c}(y)=\omega_{0} /\left(2 k_{0}\right)$, to eliminate $v_{0}(y)$ in favor of $v_{c}(y)$ where convenient. In this manner, we arrive at Eq. (4).

[1] C. Kharif and E. Pelinovsky, Eur. J. Mech. B, Fluids 22, 603 (2003).

[2] V. N. Kudryavtsev, S. A. Grodsky, V. A. Dulov, and A. N. Bol'shakov, J. Geophys. Res.: Oceans 100, 20715 (1995).

[3] R. M. Moreira and D. H. Peregrine, J. Fluid Mech. 691, 1 (2012).

[4] M. Onorato, D. Proment, and A. Toffoli, Phys. Rev. Lett. 107, 184502 (2011); V. P. Ruban, JETP Lett. 95, 486 (2012).

[5] B. S. White and B. Fornberg, J. Fluid Mech. 355, 113 (1998).

[6] D. H. Peregrine, Adv. Appl. Mech. 16, 9 (1976); D. H. Peregrine and I. G. Jonsson, Misc. Rep. No. 83-6, US Army, Corps of Engineers, Coastal Engineering Research Center, 1983 (unpublished).
[7] I. V. Lavrenov, Nat. Hazards 17, 117 (1998).

[8] I. V. Lavrenov, Wind Waves in Ocean: Dynamics and Numerical Simulations (Springer-Verlag, Heidelberg, 2003).

[9] K. B. Hjelmervik and K. Trulsen, J. Fluid Mech. 637, 267 (2009).

[10] V. Shrira and A. Slunyaev, Geophys. Res. Abs. 13, EGU20114226-1 (2011); J. Fluid Mech. 738, 65 (2014).

[11] W. J. Pierson, Jr. and L. Moskowitz, J. Geophys. Res. 69, 5181 (1964).

[12] K. Hasselmann et al., Dtsch. Hydrogr. Z. A 8(12), 7 (1973).

[13] K. Hasselmann, J. Fluid Mech. 12, 481 (1962).

[14] V. E. Zakharov, J. Appl. Mech. Tech. Phys. 9, 190 (1968). 
[15] L. H. Holthuijsen and H. L. Tolman, J. Geophys. Res.: Oceans 96, 12755 (1991).

[16] I. E. Alber, Proc. R. Soc. A 363, 525 (1978).

[17] I. Alber and P. Saffman, Tech. Rep. No. 31326-6035-RU-00, TRW Defense and Space System Group, 1978 (unpublished).

[18] E. Wigner, Phys. Rev. 40, 749 (1932).

[19] A. Hasegawa, Phys. Fluids 18, 77 (1975).

[20] Yu. Tyshetskiy, S. V. Vladimirov, and R. Kompaneets, Phys. Plasmas 18, 112104 (2011).

[21] J. E. Moyal, Math. Proc. Cambridge Philos. Soc. 45, 99 (1949); T. Takabayasi, Prog. Theor. Phys. 11, 341 (1954); B. Leaf, J. Math. Phys. 9, 65 (1968).
[22] J. R. Stocker and D. H. Peregrine, J. Fluid Mech. 399, 335 (1999).

[23] J. Rogel-Salazar, Eur. J. Phys. 34, 247 (2013).

[24] B. Eliasson and P. K. Shukla, Phys. Rev. Lett. 105, 014501 (2010).

[25] M. S. Longuet-Higgins, Proc. R. Soc. A 265, 286 (1962).

[26] H. Mitsuyasu et al., J. Phys. Oceanogr. 5, 750 (1975).

[27] D. E. Hasselmann, M. Dunckel, and J. A. Ewing, J. Phys. Oceanogr. 10, 1264 (1980).

[28] M. Onorato et al., Phys. Rev. Lett. 102, 114502 (2009); J. Fluid Mech. 627, 235 (2009).

[29] B. Eliasson, Transp. Theor. Stat. Phys. 39, 387 (2010). 